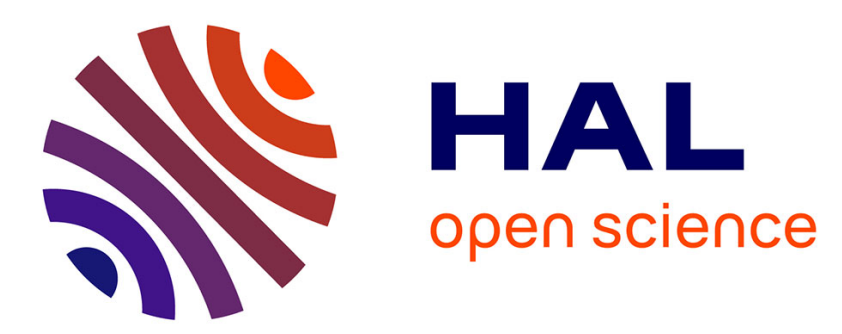

\title{
Infra-red imaging of bulk water and water-solid interfaces under stable and metastable conditions.
}

Lionel Mercury, Frédéric Jamme, Paul Dumas

\section{To cite this version:}

Lionel Mercury, Frédéric Jamme, Paul Dumas. Infra-red imaging of bulk water and water-solid interfaces under stable and metastable conditions.. Physical Chemistry Chemical Physics, 2012, 14, pp.2864-2874. 10.1039/C2CP23221A . insu-00655736

\section{HAL Id: insu-00655736 \\ https://hal-insu.archives-ouvertes.fr/insu-00655736}

Submitted on 26 Mar 2012

HAL is a multi-disciplinary open access archive for the deposit and dissemination of scientific research documents, whether they are published or not. The documents may come from teaching and research institutions in France or abroad, or from public or private research centers.
L'archive ouverte pluridisciplinaire HAL, est destinée au dépôt et à la diffusion de documents scientifiques de niveau recherche, publiés ou non, émanant des établissements d'enseignement et de recherche français ou étrangers, des laboratoires publics ou privés. 


\title{
INFRA-RED IMAGING OF BULK WATER AND WATER-SOLID INTERFACES UNDER STABLE AND METASTABLE CONDITIONS
}

\author{
Lionel MERCURY ${ }^{1, *}$, Frédéric JAMME ${ }^{2,3}$, Paul DUMAS ${ }^{2}$
}

${ }^{1}$ Institut des Sciences de la Terre d'Orléans, UMR 6113 CNRS-INSU/Université d'Orléans, 1 A rue de la Férollerie, 45071 Orléans Cedex, France.

${ }^{2}$ Synchrotron SOLEIL, L'Orme des Merisiers, Saint-Aubin- BP 4891192 Gif-sur-Yvette, France

${ }^{3}$ INRA, U1008 CEPIA, Rue de la Géraudière, F-44316 Nantes, France

"Corresponding author: lionel.mercury@univ-orleans.fr

Keywords: superheated water, micro-spectroscopy, $\mathrm{H}$-bonds networking, infra-red mapping 


\section{Abstract}

Superheated water has been studied by infrared spectroscopy to examine whether the special ability of liquid water to undergo such metastable state corresponds to the development of peculiar inter-molecular networking under tension. As the best technique to superheat water is to trap the liquid inside micro-cavities in solids (the so-called "fluid inclusions"), the role of the water-solid interfaces to stabilize the adjoining liquid is also explored with the same infra-red micro-spectroscopy tool. The key signal is the intramolecular $\mathrm{OH}$ stretching band, sensitive to the networking in the probed material. The sample of choice is liquid water occluded inside quartz cavity of micrometric size, synthetized in laboratory from pure quartz and milli-Q water. The stretching band of the superheated water shows no significant spectral difference with that of a bulk "normal" water, which means that the molecular properties of the superheating liquid is quite similar to those of the stable bulk liquid. Liquid water is readily "superheatable" but retains its "normality" under these special conditions. Additionally, this result establishes a firm ground to justify that the properties of the former are predicted extrapolating the usual (though empirical) equation of state of the latter. The infra-red signals of the water-solid interfaces are more complex. The water-solid interfaces blue-shift the signal, affecting differently the three sub-bands of the $\mathrm{OH}$-stretching. This effect was unexpected since the micro-IR spectroscopy probes volume beyond of what is classicaly assigned for the interfacial properties. In addition, the interfacial signature is clearer under superheating than with the saturation conditions, which offers an interesting (and unexpected) way to interpret the special stability of the occluded metastable water. These encouraging results give confidence on the potentialities of the high-resolution micro-spectroscopy to get insights into the molecular basis of macroscopic properties. 


\section{INTRODUCTION}

Water has one peculiar metastable state, the superheated liquid water, which is often ignored in environmental physical chemistry though it is of frequent occurrence in nature. For instance, it contributes to the surficial cycle of water through its role on the sap ascent and then on the evapotranspiration (e.g. Refs 1-2), or by favoring the water retention in nonsaturated porous materials (e.g. Refs 3-7). Despite this interest, the properties controlling the ability of water and aqueous solutions to undergo highly superheated state are not yet thoroughly understood.

Many experiments were recently performed to record the extreme tensile strength of pure water and aqueous solutions (Fig. 1), either by the Synthetic Fluid Inclusion Technique (SFIT hereafter) ${ }^{8-11}$, or by other techniques (see a recent review in Ref. 12).

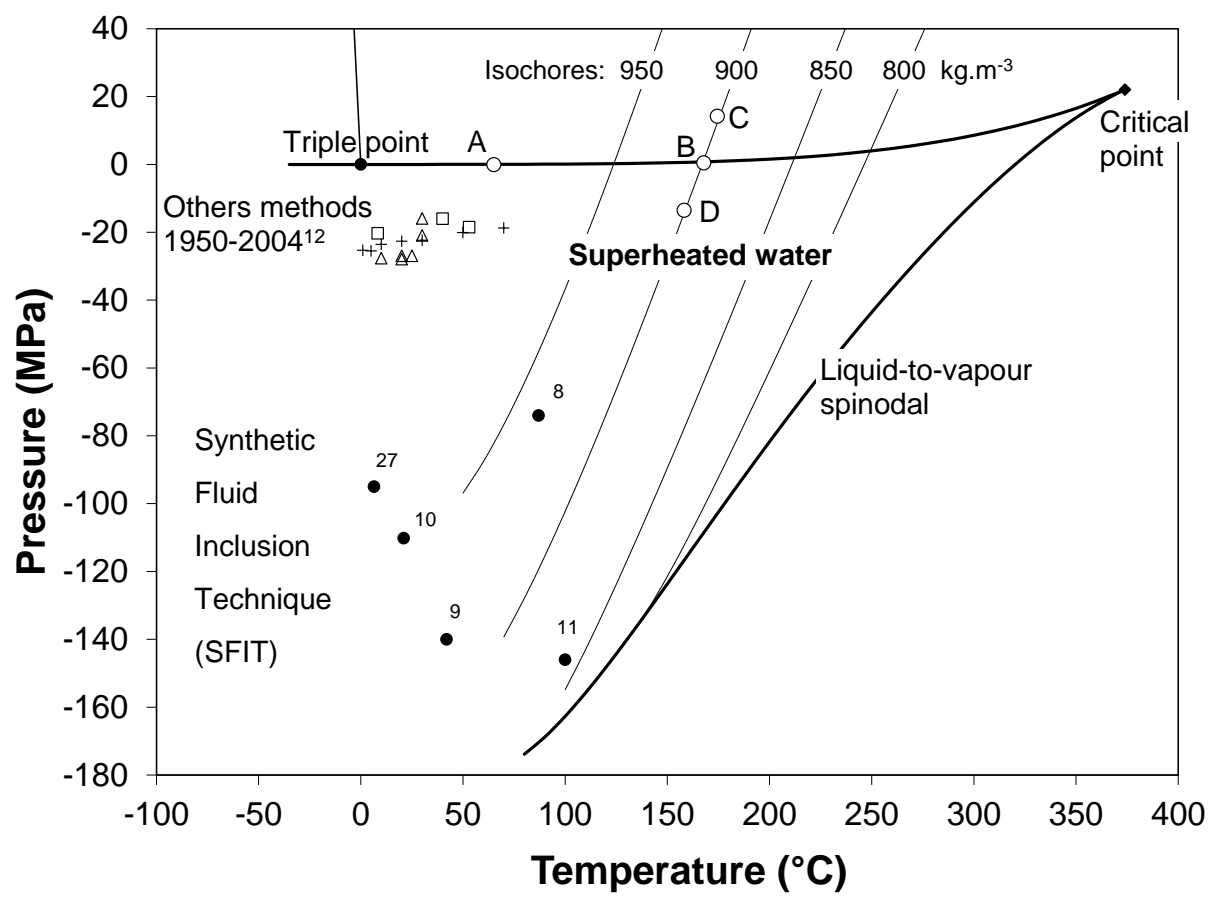

Figure 1. Phase diagram of water (IAPWS-95 equation of state ${ }^{13}$ ), extended to the superheated water domain. Experimental measurements of the extreme tensile strength are reported. The classic microthermometric path (see text) is reported from the ambient conditions $(A)$ to the homogenization temperature (Th, B), possibly up to overheating conditions (C), and cooled after down to the nucleation temperature (Tn, D) (see text). 
However, these techniques only explore the superheating limit without giving much information on the molecular features of the metastable state. We are interested in this paper to deal with the following question: is the strong cohesion revealed by the superheated water a behavior of the normal "bulk" liquid, or is it a consequence of a special molecular networking developed under the stretching conditions? In the literature, Green et al. $^{8}$ recorded the Raman spectra of an aqueous solution occluded in a fluid inclusion at $92^{\circ} \mathrm{C}$, successively superheated and stable, and found the corresponding $\mathrm{OH}$-stretching bands almost identical.

On another aspect, the SFIT is the only technique able to come close to the spinodal limit, despite that its experimental system appears less controlled and "pure" than those of the others techniques ${ }^{12}$. In particular, the solid hosting the targeted liquid in micrometric internal spaces (the so-called "fluid inclusions") certainly has crevices and surface irregularities, which are classic nucleation sites (theoretically) lowering the superheating ability. Alternatively, one could speculate that the solid walls might stabilize the trapped liquid by an adhesion effect which would make the solid-liquid contact especially robust preventing the dissolved gases and the water vapour from nucleating. In this latter case, is there a molecular signature of interface peculiarity(ies)?

The two above questions were experimentally studied by using an infrared (IR) microscope at the SMIS beamline available at the French synchrotron radiation facility SOLEIL. Using quartz samples containing fluid inclusions filled with water which we can superheat a volo, we recorded infrared spectra at the micrometric scale giving the shape and the intensity of the IR OH-stretching band inside these inclusions. The interaction of the IR light with a sampled body is an efficient and localized probe of the energetic states of this material. Specifically, the $\mathrm{OH}$ stretching band probes the intra-molecular motions of water molecules which are sensitive to the hydrogen bonds networking. Therefore, this is a tool well adapted to probe the molecular cohesion, and to test the arrangement characteristic of the sample of interest. 
We measured the IR spectra of superheated and stable water and compared the profile of the $\mathrm{OH}$ band throughout a fluid inclusion (IR map). We aim to see if some band shift(s) and/or deformation(s), especially at the water-solid interfaces, could account for the special stability of the liquid water inside these materials. This study is exploiting the high spatial resolution of the IR micro-spectroscopy enhanced by the brilliance of the synchrotron source.

\section{SAMPLE AND PROTOCOL}

\subsection{Quartz sample}

The SFI quartz samples hosting biphasic water-filled inclusions were synthetized in internally-heated pressure vessels (IHPV, or "gas bombs"). The hydrothermal synthesis was performed either at $300 \mathrm{MPa}$ and $626^{\circ} \mathrm{C}$ during 4 days (sample 1), or at $750 \mathrm{MPa}$ and $600^{\circ} \mathrm{C}$ during 13 days (sample 2). The IHPV is loaded with Au-capsules filled with a (millimetric) piece of quartz, the selected liquid (pure water in the two samples) and amorphous silica. The solid quartz is first thermally cracked before being put in the capsules with the imbibing liquid. It will be healed at high P-T conditions owing to the presence of amorphous silica in the capsule.

The hydrothermal healing restores a massive solid which contains however what is classically termed "fluid inclusion" (Fig. 2), that is cavities (5-50 $\mu \mathrm{m}$ size) containing the liquid which was present in the synthesis capsules. Once the required synthesis time elapsed, the power supply was turned off and the IHPV was allowed to cool down to the room temperature before be opened to recover the capsules. After cutting and (doubly) polishing the quartz seeds, the final thickness of samples is around $500 \mu \mathrm{m}$, enabling an easy observation of the included fluid inclusions with classic microscopy (Fig. 2).

The hydrothermal process could favour the dissolution of silica from the quartz matrix inside the cavity, modifying the original purity of the occluded liquid. However, the possibility of significant leaching was ruled out by the absence of $\mathrm{Si}-\mathrm{OH}$ bands in the recorded spectra. 
A.

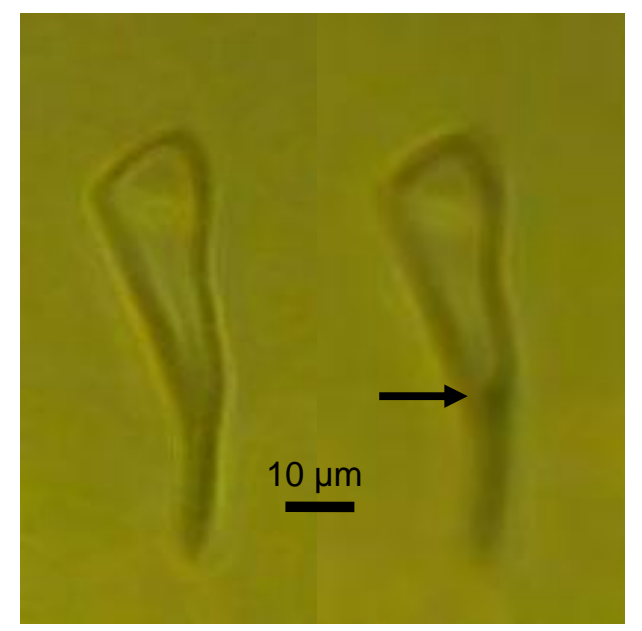

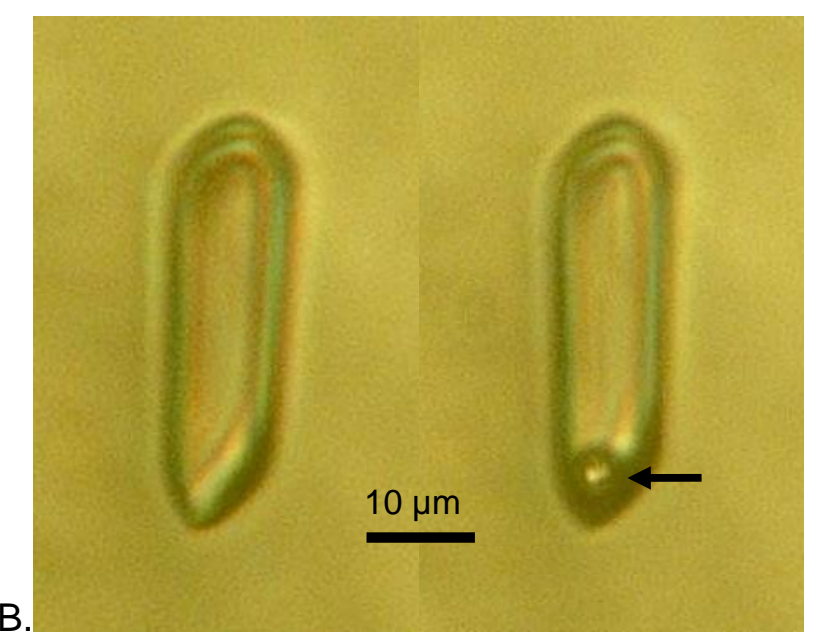

B.

Figure 2. Photographs of the sampled fluid inclusions (A. sample 1; B. sample 2). On the left of each sub-figure, the monophasic inclusions contain the superheated liquid. To the right, the same inclusions after nucleation (biphasic mixture), the arrows point to the visible bubble. These samples offer many advantages for the targeted experiments. First, the total size of each sample makes easy to manipulate it, and also the size of the fluid inclusions enables to map precisely the liquid-only, solid-only and interfacial locations. Meanwhile, the water volume is significant enough to get signals significantly above the background. The second great advantage of these materials is that the superheating conditions are easily measured and controlled, with a reproducible cooling-heating process (microthermometric procedure, see below).

\subsection{Experimental set-up}

The IR experiments were performed at SOLEIL Synchrotron (Gif sur Yvette, France) at the SMIS beamline. The average current in the storage ring was about $400 \mathrm{~mA}$. The beamline is equipped with a Continuum XL microscope (Thermo Fisher Scientific, USA) coupled to a Nicolet 5700 FT-IR spectrometer (Thermo Fisher Scientific, USA). The microscope comprises a liquid nitrogen cooled mercury cadmium telluride (MCT-A) detector $(50 \mu \mathrm{m})$ and we used in this work a $32 x$ infinity corrected Schwarzschild objective $(N A=0.65)$ and a matching $32 x$ condenser. This objective offers to work in confocal mode, specifically collecting the transmittance signal from a given plane $(2-3 \mu \mathrm{m}$ thick, equal to the IR 
wavelength) located at a precise depth inside the sample. This functionality is especially interesting in our "interface-focused" experiments, eliminating the influence of the other interfaces possibly crossed by the beam along the whole thickness of the sample. All spectra were obtained using a double path single masking aperture size of $8 \times 8 \mu \mathrm{m}$. The spectra were collected in the $6000-2000 \mathrm{~cm}^{-1}$ mid-infrared range at a spectral resolution of $4 \mathrm{~cm}^{-1}$ with 64 co-added scans. The profiles were calculated by using the OMNIC software (Thermo Scientist, USA). The term "profile" is used to refer to the integrated spectral intensity of a specified spectral region for each sample point.

The thermal changes in the inclusion required to produce the superheating conditions are afforded by a heating-cooling stage Linkam IR-compatible (FTIR 600 stage). The sample is put on a silver block whose temperature is precisely controlled $\left( \pm 0.1^{\circ} \mathrm{C}\right)$ along the heating and cooling ramps. To avoid thermal gradients, the sample is capped with a silver cover which has already revealed very efficient ${ }^{11}$. The micro-thermometry technique enables to put the trapped liquid into the superheating state. Actually, the procedure (Fig. 3) consists of the progressive heating with the Linkam stage of one sample, in general containing initially the biphasic liquid+vapour assemblage $(\mathrm{L}+\mathrm{V})$. The first step corresponds to the displacement along the saturation line, with a progressive change of temperature, liquid density and bubble pressure ( $A$ to $B$ path, Fig. 1). At a particular density, the trapped liquid invades the whole inclusion space: there is no more vapour and the inclusion is said to be homogenized ( $B$ point, Fig. 1). The temperature at which this filling appears is then the homogenization temperature, noted Th. Further heating drives the P-T conditions in the inclusion along the isochore inside the stable domain of liquid (B to $C$ path, Fig. 1). The second step is the progressive cooling of the sample, which follows the isochoric path as long as the inclusion remains homogeneously filled with liquid ( $C$ to $D$ path without nucleation at $B$, Fig. 1). The temperature of the bubble appearance is called the nucleation temperature $(\mathrm{Tn})$ and is always located within the tensile domain: nucleation in fluid inclusions always disobeys the saturation conditions. 
The two inclusions studied below have reproducible homogeneization $\left(\operatorname{Th}_{\text {SAMPLE1 }}=\right.$ $\left.324.5^{\circ} \mathrm{C} \pm 0.5^{\circ} \mathrm{C} ; \quad \mathrm{Th}_{\text {SAMPLE2 }}=167.7^{\circ} \mathrm{C} \pm 0.5^{\circ} \mathrm{C}\right)$ and nucleation temperatures $\left(\mathrm{Tn}_{\text {SAMPLE } 1}=\right.$ $309.3^{\circ} \mathrm{C} \pm 0.1^{\circ}$; and $\left.\operatorname{Tn}_{\text {SAMPLE } 2}=115.5^{\circ} \mathrm{C} \pm 0.8^{\circ}\right)$. These values are low enough to work with the Linkam stage directly put on the IR microscope stage $\left(T \leq 350^{\circ} \mathrm{C}\right)$. Also, the lifetime of superheated water at $313^{\circ} \mathrm{C}$ (sample 1) is 6 minutes, long enough to record the IR map. According the IAPWS-95 equation of state ${ }^{13}$, the occluded liquid has a density of 655.64 $\mathrm{kg} / \mathrm{m}^{3}$ (saturation density at $324.5^{\circ} \mathrm{C}$ and $11.97 \mathrm{MPa}$ ), and undergoes at $309.3^{\circ} \mathrm{C}$ a tension amounting to -0.62 bars, and to $2.41 \mathrm{MPa}$ at $313^{\circ} \mathrm{C}$. As for the sample 2, the lifetime at $117^{\circ}$ is 4 minutes, the superheated liquid is characterized by: $P_{\text {liquid }}=-78.5 \mathrm{MPa}$ at $117^{\circ} \mathrm{C}$, with a density amounting to $899.8 \mathrm{~kg} / \mathrm{m}^{3}$. It is interesting to notice that the sample 2 display similar $(P, T)$ pair than that observed by Green et al..$^{8}$ which was $\left(-80 \mathrm{MPa} ; 92^{\circ} \mathrm{C}\right)$.

\subsection{Protocols}

First, the $\mathrm{OH}$-stretching band was recorded on the monophasic inclusion at the same location, at different temperatures along the monophasic path. We aim at comparing the stable/metastable IR signals: any shifts or deformation in the bands would be related to a difference in the molecular networking. Second, the inclusion was brought back to the stable biphasic state (water-vapor system), once the bubble nucleated.

In these protocols, the $\mathrm{OH}$-stretching band was recorded over the whole xy plane, with a special interest to the different interfaces visible (water-solid, water-air when existing) in the sampling zone. As already outlined, the confocal signal is collected along a micrometric thickness around the nominal depth, discarding a significant influence of other interfacial structures above or below the sampled plane. For instance, the top and down interfaces compulsory crossed by the light at the entrance and the exit of the solid cavity, do not contribute to the recorded spectra. Also, we carried out a mapping of the plane surface by incremental displacement of the $8 \times 8 \mu \mathrm{m}$ beam (see the aperture size above). The whole surface is explored by this square records successively processed side-by-side, and enabling to follow the IR contrast/similarity between the interfacial sites and the bulk parts of 
the inclusion. As developed below, our protocol bears on the idea that the signal taken at the borders of the inclusion magnifies an-otherwise invisible signature, having a longer pathlength (equal to the IR plane thickness) to sample the interfacial effects.

The two samples explored in this study are close to each other in terms of composition (quartz + water), and synthesis conditions (see above), but are significantly different in terms of geometry. Thus, the inner surface state (roughness, irregularities, ...) should be similar, since it depends upon the synthesis conditions (time and $(\mathrm{P}, \mathrm{T})$ pairs) which are close to each other. By contrast, the global shape is irregular for the sample 1 and regular for the sample 2, implying that the quantity of wedges, crevices, or corners could be very different from the 1 to the 2 sample. At last, the intensity of the superheating varies much between the two: the liquid trapped in the sample 1 is oscillating between a weakly negative and positive superheating pressure, while that contained in the sample 2 shows a strongly negative superheating pressure.

As a conclusion, our protocols consist in comparing the absorption of IR light by the same liquid, contained in the same inclusion (at constant geometry, surface irregularities, ...), at almost the same temperature, but at two different internal pressure (superheating vs. saturation pressure). It is why we expect that the conclusions gained in these two different samples, if similar, only refer to the role of the superheating, with a special attention to the interfaces role, whatever the local peculiarities.

\section{RESULTS AND DISCUSSION}

A first approach of the global signal can be made by mapping the absorbance maximum in the two samples, before and after the nucleation (Figs 3-4). 


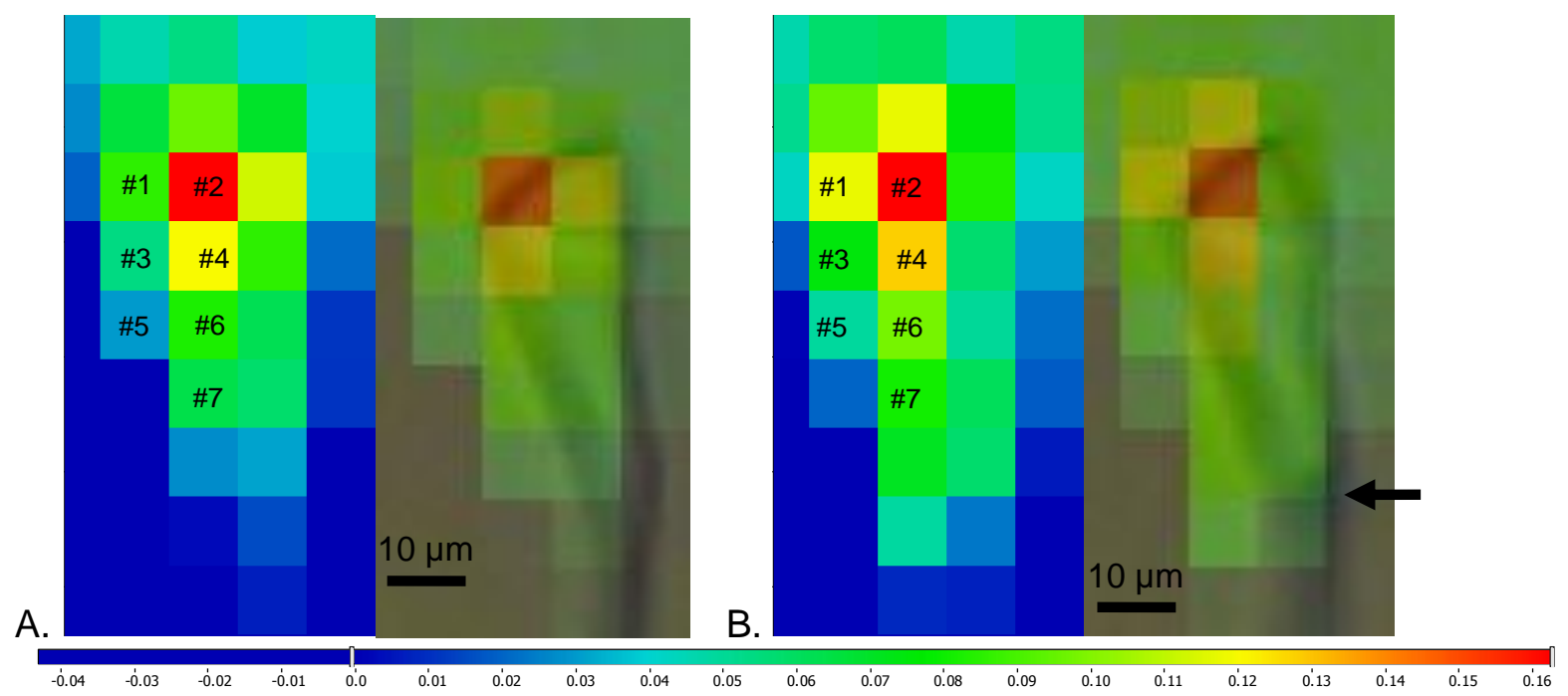

Figure 3. IR map of the sampled inclusion in the sample 1, before (3A) and after (3B) nucleation. Each square scales the beam size $(8 \times 8 \mu \mathrm{m})$ over each recorded spectrum, and the colour relates to the absorbance scale, taken at the main peak of the $\mathrm{OH}$-stretching band: $3640 \mathrm{~cm}^{-1}$.

In figure 3 (sample 1), the spectra that were numbered are those absorbing (at the $\mathrm{OH}$ stretching band) more than 0.05 (abs. u.). Unfortunately, the bubble area (see arrow) and in general the downside part of the inclusion gave no signal, and so the feed-back of the bubble (water-air interface) on the IR signal cannot be studied. As a whole, the absorbance appears more intense after the nucleation in the main part of the inclusion, notably in the downside part (around the bubble, Fig. 3).

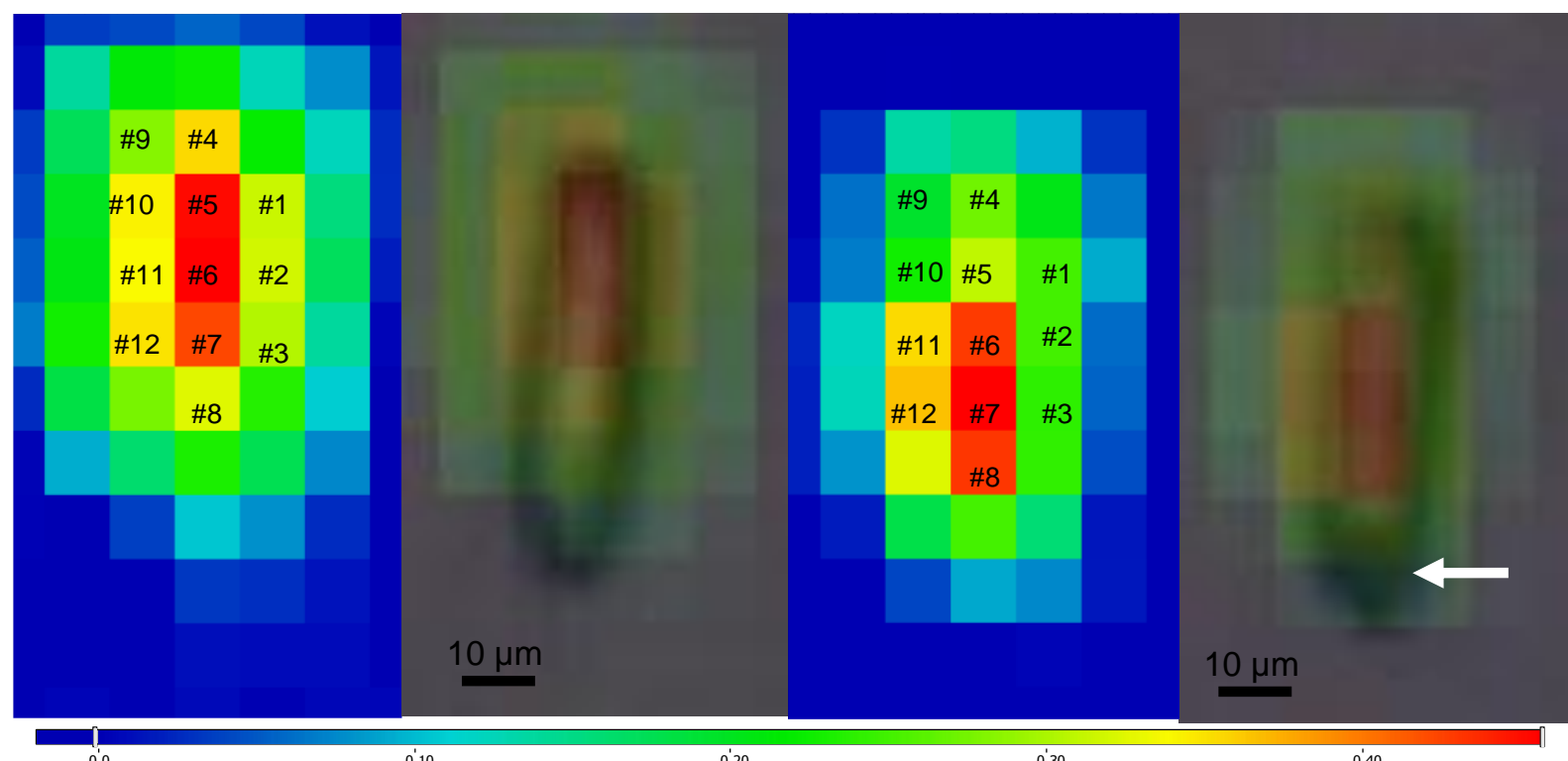


Figure 4. IR map of the sampled inclusion in the sample 2, before (two left photos) and after (two right photos) nucleation. Each square scales the beam size $(8 \times 8 \mu \mathrm{m})$ over the recorded spectrum, and the colour relates to the absorbance scale, taken at the main peak of the $\mathrm{OH}$ stretching band: $3570 \mathrm{~cm}^{-1}$.

On figure 4, the IR map of the sample 2 appears visibly shifted to the right when turning from the monophasic (left) to the biphasic (right) inclusion, like the sample 1. The main peak of the band is located at a smaller wavelength $\left(\Delta \omega=70 \mathrm{~cm}^{-1}\right)$ which relates to the difference in the temperature range $\left(310^{\circ}\right.$ vs. $\left.110^{\circ} \mathrm{C}\right)$. This interval is consistent with the $0.25-0.50 \mathrm{~cm}^{-1} /{ }^{\circ} \mathrm{C}$ shift already observed in saline fluid inclusions along similar thermal range ${ }^{30}$.

At first sight, the absorbance signal appears more pronounced (hot colours) with the monophasic fluid than with the biphasic one, in the special case of the IR signal taken along the borders of the inclusion. At these locations, the absorbance reddens before than after the nucleation, while the three red squares moved to the bottom, toward the bubble.

As a first conclusion, it appears that the IR signal is changing when the nucleation occurs, and the changes seem different when close or away to the water-solid interfaces.

\subsection{Stable/metastable water}

\subsubsection{Bulk signals}

The spectral difference between the spectra of the superheated water and that recorded at the same location but after nucleation of the bubble was appreciated by plotting each two records on the same graph (Figs 5-6: dark blue: monophasic liquid; light blue: biphasic liquid). The baseline was drawn over the $3800-3150 \mathrm{~cm}^{-1}$ interval for the spectra of the sample 1, and the $3700-3150 \mathrm{~cm}^{-1}$ interval for the spectra of the sample 2. Also, the red curves on figures 5 and 6 give the (monophasic-biphasic) subtraction using the two baselinecorrected spectra. The subtraction is calculated (omnic software) data point by data point, and algebraically, it works like this: Sample - Reference $x$ Factor $=$ Result. The "sample" is always the liquid taken in the monophasic state and the "reference" is the one in the biphasic state. The "factor" is automatically calculated to scale the intensities of the two spectra, so 
Revised version of the Manuscript ID CP-ART-10-2011-023221. Submitted to Physical Chemistry Chemical Physics. December 14 2011.

that to magnify the spectral difference in the $\mathrm{OH}$-stretching band.In the sample 1 (Fig. 5), the spectrum under superheating is taken just before nucleation at $313^{\circ} \mathrm{C}$, while the stable liquid spectrum is taken after having cooled the sample slightly below $\mathrm{Tn}\left(309^{\circ} \mathrm{C}\right)$ before resetting its temperature at $310^{\circ} \mathrm{C}$.


spectrum taken at $310^{\circ} \mathrm{C}$

spectrum taken at $313^{\circ} \mathrm{C}$

result of the spectra subtraction

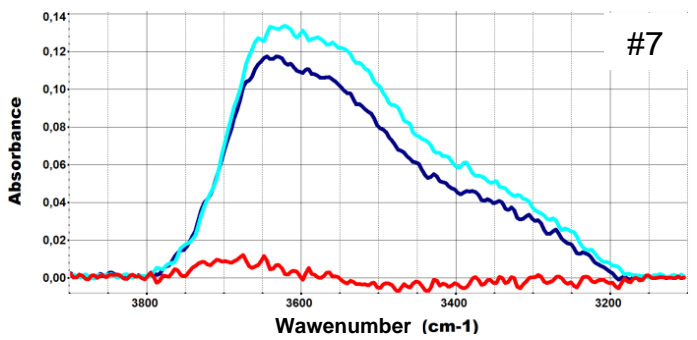

Figure 5. Baseline-corrected spectra of metastable superheated (at $313^{\circ} \mathrm{C}$ ) and stable water (at $310^{\circ} \mathrm{C}$ ) in the sample 1. The numbers relate to the location on the map (see Fig. 3).

A slight increase of the absorbance is visible on most of the biphasic spectra $\left(310^{\circ} \mathrm{C}\right.$, light blue) when at the borders of the inclusion (\#1, \#3, \#5, \#7). That suggests that the stretching signal is less intense at the interfaces under the superheated state, which may indicate that the water-solid interactions are stronger with the superheated liquid than with the stable one. 
Revised version of the Manuscript ID CP-ART-10-2011-023221. Submitted to Physical Chemistry Chemical Physics. December 14 2011.

Meanwhile, the spectra taken in the thicker part of the inclusion $(\# 2, \# 4, \# 6)$ appear almost the same before and after the nucleation. In particular, the shape of the band (see the horizontality of the red subtraction line) does not change between the two (before/after) what indicates that the energetic features of the water in the two states (monophasic superheated / biphasic stable) are similar. In other words, the superheated liquid occluded in a monophasic inclusion, retains the same properties as the stable liquid trapped in a biphasic inclusion, despite the two liquids have not the same internal pressure $\left(\Delta \mathrm{P}=\mathrm{P}_{\text {biphasic }}-\right.$ $\mathrm{P}_{\text {monophasic }}=7.3 \mathrm{MPa}$ ). Our record indicate that far from any interfaces, the superheated metastable liquid and the heated stable one absorb IR light similarly along the 3800-3000 $\mathrm{cm}^{-1}$ range, which means they are similar (vibrational) properties. That gives firm ground to state that, even metastable, the superheated water is classic normal water, obeying the equation of state. Meanwhile, the interfacial signature is clearly marked by an increase of the absorbance (light to dark blue), and a change in the band shape (red curve). Adopting the same line of reasoning as just before, we can feel that these differences correspond to the presence of properties along interfaces different from those in the thicker parts of the inclusions.
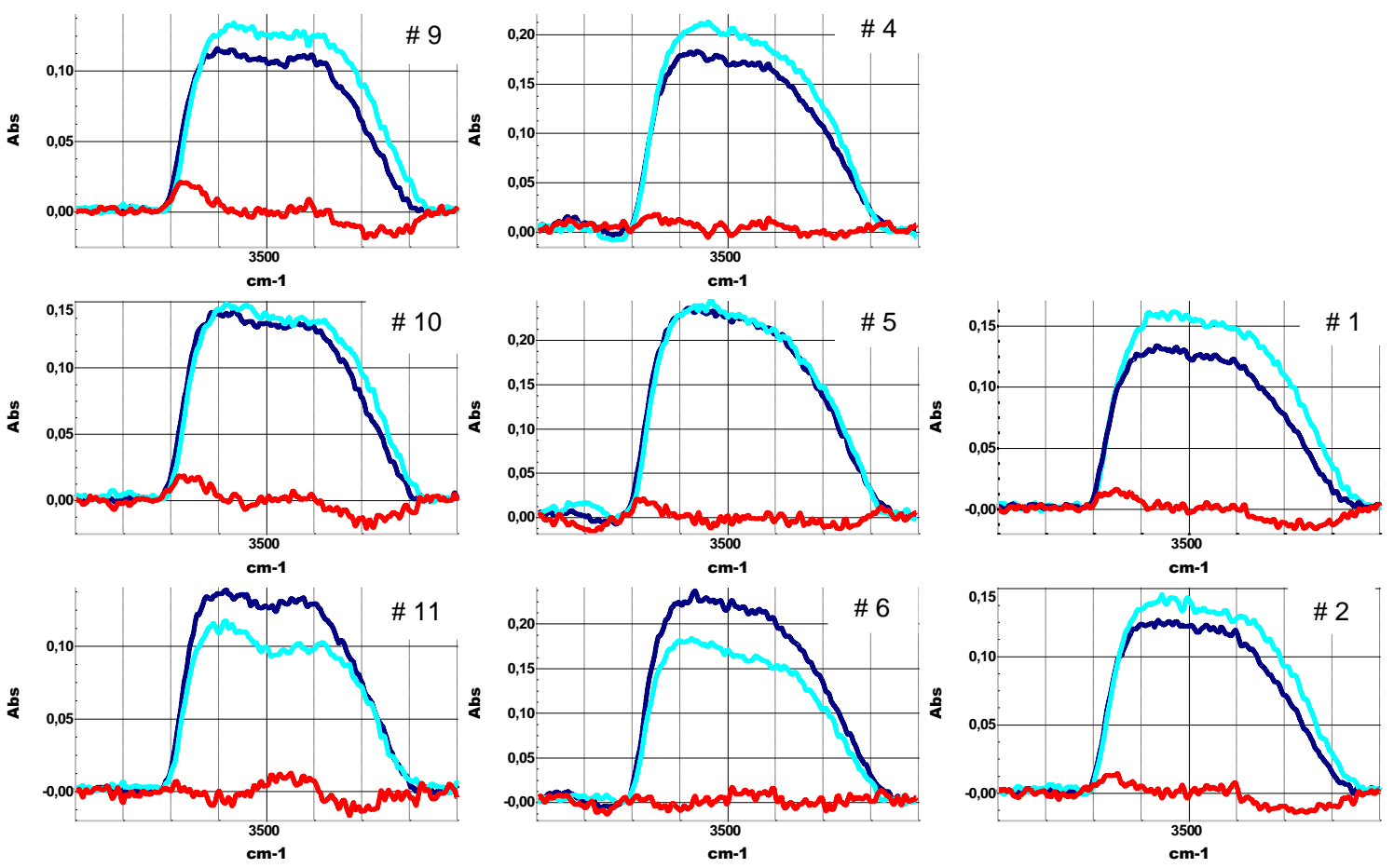

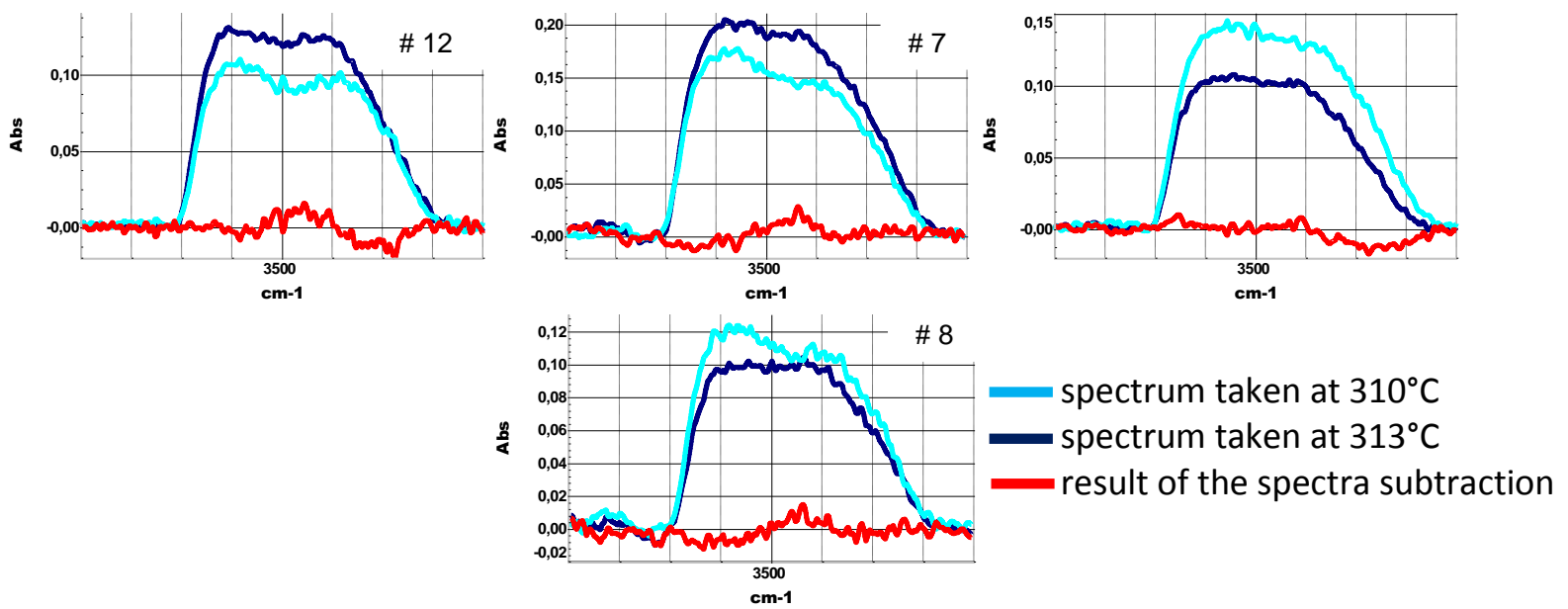

Figure 6. Baseline-corrected spectra of metastable superheated (at $117^{\circ} \mathrm{C}$ ) and stable water $\left(\right.$ at $110^{\circ} \mathrm{C}$ ) in the sample 2. The numbers relate to the location on the map (see Fig. 4).

When moving to the second sample (Fig. 6), the signals interpretation appears more complex: the before/after \#5 spectra absorbs similarly, but the red curve is clearly not horizontal, meaning that the band shape changed. The \#6 shows an almost horizontal red line (constant band shape) but with a decreasing absorbance after nucleation. This fact means that there is only a decreasing quantity of liquid sampled by the beam at constant location and conditions, except the nucleation event. We propose the presence of tiny vapour bubbles in the field to account for this decreasing liquid amount. Only one large bubble is visible on the photo (Fig. 4), but it is current to have rings of tiny bubbles aligned along the water-solid interfaces ${ }^{11}$. Actually, the nucleation is always heterogeneous in the inclusions, classically favoured by the irregularity of the wall surfaces which become bubbles-rich, especially when the boiling is very large due to the high degree of superheating, like in the present sample.

As a whole, two trends can be extracted from this sample. The first is the previouslydescribed interfacial signature with a lower absorbance for the superheated liquid compared to the stable one: \#1 to \#4, \#9 and possibly the \#8 and \#10. The second shows the opposite, with the superheating liquid absorbing more than the stable one, for the \#6-7, and \#11-12. At first sight, this could be related to a decreasing quantity of sampled liquid, possibly due to the presence of vapour bubbles. The \#5 (same absorbance), and \#6 (same shape) give ground 
that the two liquids can be compared, and that the spectral differences otherwise recorded translate energetic differences linked to the water state.

As an intermediate conclusion, we may propose that the absence of spectral difference between the metastable and stable liquid (when no interface significantly acts) indicates that no noticeable changes occurred when the liquid enters a metastable state, in terms of the dynamics of the molecular network. And that conclusion is met when the saturation-tospinodal distance is small (high temperature, sample 1), or large (low temperature, sample 2). As a consequence, the properties of the metastable liquid water can be suitably predicted by extrapolating the equation of state of bulk water from the P-T stable conditions down to the superheated P-T domain. By that conclusion, the spectroscopic signature supports well the extrapolative tests performed on the equation of state towards the metastable domains $^{13,15-16}$. We can also notice that it was the findings outlined by Green et al. ${ }^{8}$ through Raman studies.

\subsubsection{Band decomposition}

In order to go beyond the simple qualitative trends, we performed a decomposition of the $\mathrm{OH}$-stretching bands into its sub-bands, to explore how they evolve through the previously detected changes. The change in the shape and the main peak of the band itself, directly observed by eyes, can be more quantitatively examined by looking at the behaviour of each of these sub-bands when the liquid turn from the metastable state to a stable state, and/or when probing the interface or the bulk.

The $\mathrm{OH}$ stretching band is classically composed of three (at least) sub-bands assigned to different populations of water molecules (e.g. Ref. 14): $3295 \mathrm{~cm}^{-1}$ for the tetrahedrallybonded molecules (the "ice-like" band); $3460 \mathrm{~cm}^{-1}$, whose position corresponds to a water structure more or less distorted because the $\mathrm{H}$-bond coordination number is lower than 4; $3590 \mathrm{~cm}^{-1}$ is ascribed to the poorly connected molecules (the "vapour-like" band).

The decomposition was done under the Igor software using the "multipeak fitting" tool. The three above values were loaded, each sub-band was ascribed a gaussian shape, while the 
Revised version of the Manuscript ID CP-ART-10-2011-023221. Submitted to Physical Chemistry Chemical Physics. December 14 2011.

three characteristic parameters (location of the main peak, height, width at half height) were let free to find the best solution (minimal $\mathrm{X}^{2}$ ).
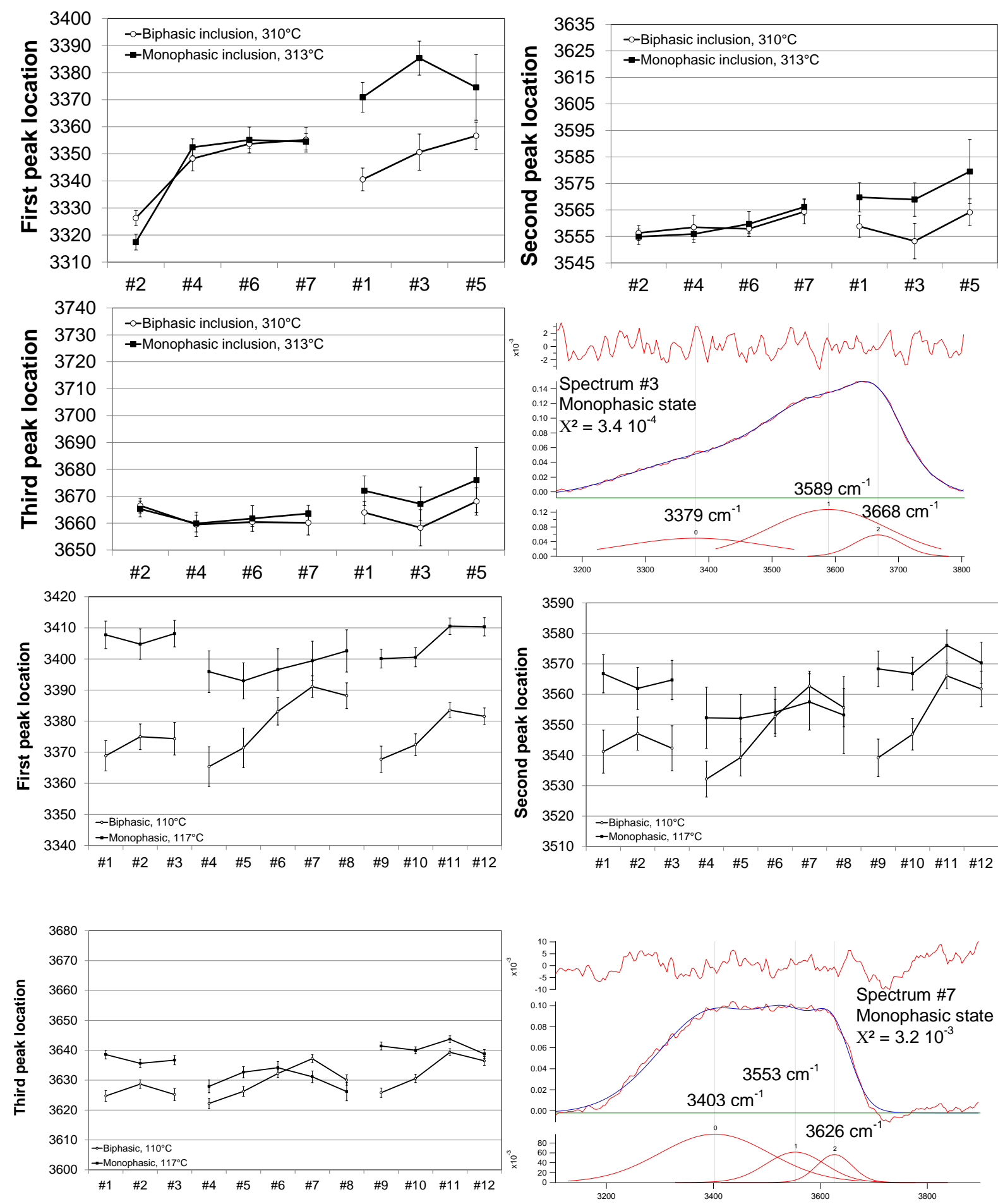

Figure 7. Decomposition of the $\mathrm{OH}$-stretching band into three sub-bands (see text), for the samples 1 (four upper graphs), and 2 (four lower graphs). All the ordinates axis are plotted over the same $90 \mathrm{~cm}^{-1}$ interval. 
The sample 1 gives the simplest results: the sub-bands are quite the same between the superheated and the stable liquid when the spectra are taken in the thicker parts of the inclusion (\#2, \#4, \#6, \#7). However one can note the similarity of all the sub-bands at the \#7 location whereas the two global $\mathrm{OH}$ bands were separated (see Fig. 5). The spectra evidencing the interface effect $(\# 1, \# 3, \# 5)$ are all probed along the same transect (see Fig. 3). The spectral shift of the superheating affects the three sub-bands, more particularly the first "ice-like" peak: the monophasic liquid deviates systematically by $10-30 \mathrm{~cm}^{-1}$ from the biphasic liquid, making to suppose that the water-solid interactions are specific under superheating. As a whole, the monophasic metastable liquid absorbs less the IR light (see above), and is blue-shifted with respect to the biphasic stable liquid, when the spectra is taken at the borders of the inclusion.

In the case of the sample 2, the sub-bands are less regularly arranged, but again this is the first "ice-like" peak which puts best the effects into light. Five spectra (\#6, \#7, \#8, \#11, \#12) have their constituting sub-bands plotted at similar wavelength between superheated and stable liquid, except the first sub-band of the \#11 and \#12, strongly shifted toward the longer wavelength (to the "blue"). Also, this crude similarity could be discussed, since in detail the spectra are not identical through the three sub-bands. These differences are small enough to avoid over-interpret them, just could be outlined that the temperature and pressure differences between the two liquids $\left(\Delta \mathrm{T}=7^{\circ} \mathrm{C} ; \Delta \mathrm{P}=78.6 \mathrm{MPa}\right)$ might be accompanied by a slight relaxation of the water structure. A qualitative indication is the molar volume of liquid water which is $18.94 \mathrm{~cm}^{3} / \mathrm{mol}$ at $\left(110^{\circ} \mathrm{C}, 0.14 \mathrm{MPa}\right)$ and $20.02 \mathrm{~cm}^{3} / \mathrm{mol}$ at $\left(117^{\circ} \mathrm{C},-78.5\right.$ $\mathrm{MPa})$, a quite small difference in any case.

The other spectra (\#1 to \#5, \#9, \#10) of the superheated liquid are shifted toward the longer wavelength (to the "blue") with respect to the stable liquid, and that whatever the sub-band. These locations are those along the water-solid interfaces, and therefore the present trend is consistent to what has been concluded with the sample 1: superheated water is more blueshifted at the interface than the stable liquid. That makes to suppose a special molecular arrangement at the interface under superheating as previously observed. Yet, it is clear that 
the shift between the monophasic and the biphasic inclusion is comparatively more pronounced in this inclusion 2 than with the previous 1 one.

It is the right place to recall the specificity of this present inclusion when in biphasic condition. According to the global band (previous paragraph), we suspected the presence of tiny bubbles along the water-solid interfaces. That assumption may account for the present large variation in the wavelength of the first and second sub-bands, more or less red-shifted as a function of the location. To see clearly this fact, it is important to fix the reference to the three (\#6, \#7, \#8) spectra displaying similar sub-bands before and after nucleation. This crossing behaviour establishes that somewhere inside the inclusion, the two liquids are similar, as previously argued. As a consequence, the other spectra are read by comparison to these references, and then appear obviously and variably red-shifted. The fact that the shift is gradually less pronounced from the first (the ice-like band) to the third sub-band (the vapourlike band) is a consistent indicator that the acting effect may have a vapour-like signature.

The two above paragraph can be synthetized by stating that the comparative blue-shift of the superheating liquid along the interface is magnified in the sample 2 , by a simultaneous redshift of the liquid hosted by the biphasic inclusion, also along the interfaces. These interfacial opposite effects, discussed more extensively below, should not overshadow the evidence that the superheated and the stable liquids display the same energetic profile when not affected by others effects.

As a conclusion, the decomposition of the main band confirms the previous estimates. First, the metastable and stable liquids do not differ in terms of energetic features, when recorded at similar temperature. This experimental fact justifies that the properties of the superheated liquid be calculated using the classical equation of state, adapted to describe the "normal" stable water. Second, the water-solid interfacial signature shows features specific of the water state: the IR absorption is different with the superheated liquid, tending to indicate that the interfacial contact is energetically different. Third, the water-solid and water-air interfaces have an IR signature, especially visible on the "ice-like" peak $\left(3295 \mathrm{~cm}^{-1}\right)$ in the case of the water-air interface, at the sample scale. This fact was not really expected since the classical 
Revised version of the Manuscript ID CP-ART-10-2011-023221. Submitted to Physical Chemistry Chemical Physics. December 14 2011.

thickness of one interface through high resolution methods, is around 1-5 nm, a size normally not sensitive with IR micro-spectroscopy.

\subsection{Effect of the water-solid interfaces}

The IR response ascribed to the water-solid interfaces can be precisely examined by following the spectral changes in the same inclusion from the center to the periphery, under the same conditions and materials.

\subsubsection{Superheated liquid occluded in monophasic inclusion}

The first trend can be observed on sample 1 in the figure 6 , by observing how the sub-bands evolve when moving vertically.

The vertical displacement towards the borders of the inclusion from \#2 to \#7, corresponds to an increase of the first peak from the \#2 to the three others (\#4-6-7), which display nearly the same profile: these three are most probably all affected by the interface, and the interfacial signature itself is a shift towards higher wavenumber (to the "blue"). On the other vertical, the \#1-3-5 spectra show a enhanced blue-shift than for the \#4-6-7, this time affecting (differently however) the three sub-bands. These data point to a variable weight of the IR signature of the water-solid interface on the (mainly) first peak of the band, depending on the respective locations of the beam and the interface.

A close observation of the monophasic data in the sample 2 results bring to the same conclusion, despite the large differences between the two samples. The spectra taken away from the solid walls, show the sub-bands absorbing at a smaller wavelength than those taken along the borders. The first and second sub-bands seem more affected than the third, but the signal is globally blue-shifted like in the sample 1. If retaining the blue-shift as an interfacial signature, and following the second and third sub-bands, it appears that the \#1 to \#3, and \#9 to \#12 spectra are all affected by the interface. However, the behaviour of the first sub-band does not give the interfacial signature to the \#9 and \#10 spectra which should be bulk-like. That contrasted conclusion evidences the fact that the signature here deduced certainly 
echoes a complex interplay between the respective location of the beam and the interface, but also echoes the local geometry of the probed interface.

At this stage, we can outline again that the detectability of the interfacial effect at the sample scale was not at all expected, because the water-solid interfacial thickness is generally supposed to be around the nanometre. The sub-band with the highest wavenumber is classically ascribed to poorly connected molecules, especially those originating from dimers with linear bonds $\left(\omega_{\text {dimer }}=3640 \mathrm{~cm}^{-1}\right)^{17}$. In parallel, we can outline that the interfacial $\mathrm{H}_{2} \mathrm{O}$ molecules are linked to the framework but not bonded to any other molecules ${ }^{18}$ resulting in a poor connection to the local network. As a consequence, the blue-shift of the global band towards higher wavenumber is therefore ascribed to the characteristics of the water-solid interface along which the water structure is disrupted (chaotrope or structure-breaker effect). The band decomposition demonstrates that the present reasoning is ignoring other parameters controlling the behaviour of the sub-bands which are not equally blue-shifted, possibly due to the local geometry.

As a whole, this conclusion meets the neutron scattering study of water confined in GelSil ${ }^{19}$ which show that the confinement induces strongly destructive effects on the intermolecular network of interfacial water. A complementary study using vibrational spectroscopy ${ }^{20}$ demonstrated that this structure-breaker effect is not always retrieved and depends on the confining materials: the zeolite-water interactions are structure-maker. Another possible effect is the decreasing density of water at the vicinity of certain hydrophobic surfaces presently referred to the development of a vapour-like film ${ }^{21-22}$. According to our previous reasoning, we can safely conclude that the IR signal points to a structure breaker effect of the water-solid interfaces on the water network.

At first sight, this result may be surprising since classically the structure state could be associated with the level of the molecular cohesion, so that a structure-broken liquid should be expected less stable than the bulk. However, an ambiguity emerges from dealing with the water structure without a partial view of the global picture. In particular, the role of the silanol $\mathrm{Si}-\mathrm{OH}$ bonds, the exact number and geometry of the hydrogen bonds cannot be completely 
explored with our present approach focused on the $\mathrm{OH}$-stretching band, though these parameters may contribute to the final cohesion. Among others, the interfacial effect has been linked to a directionality of the bonds at the interface ${ }^{23}$, a proposition which cannot be suitably discussed with our results. The only non-ambiguous result we may propose is that the water-solid interfacial IR structure under superheating is different than under the saturation conditions, which may participate in the over-stability of the occluded liquid pointed out in the introduction.

However, the most surprising point is that we were able to record that effect in a continuously filled material with a $8 \times 8 \mu \mathrm{m}$ beam in a transmittance mode. Actually, it is classically admitted that the interface effect is 2-3 molecular layers thick. Our hypothesis is that the water-solid contact is sufficiently vertical for the probing light to record the (weak) interfacial signal along the total thickness of the inclusion allowing the longer pathlenght to sample the effect. It is here worthwhile to remind that the light is $8 \times 8 \mu \mathrm{m}$, but not all the light probes the water, since part illuminates out of the cavity. Eventually, this proposition is somewhat speculative but enables us to reconcile the observed blue-shift with the well-established fact of the nanometric thickness of the interface.

\subsubsection{Stable liquid occluded in biphasic inclusion}

The spectra were recorded at the same xy locations and following the same protocol as with the superheating conditions. The temperatures are close the same, only the internal pressure of the occluded liquid is supposed to vary, turning from the pre-nucleation pressure to the saturation one after boiling.

Inside the sample 1 , the reference spectrum is that at the \#2 location which displayed the bulk-like behaviour, more exactly the behaviour the less affected by the interfacial blue-shift (we have no real bulk reference). The first sub-band shows the same evolution as previously when one get farther to this bulk position: a shift toward longer wavelength. Most of the others spectra are identical, even when decomposing the band into its three sub-bands: the three sub-bands of the \#3 to \#7 align in a quasi-straight line, inside the error bars. Among 
these "other" spectra, the \#1 appears specific, showing a shift intermediate between the bulklike behaviour (that shown by \#2) and the interfacial one.

Also worthwhile is the "normality" of the \#1 to \#3 vertical, with no more the additional blueshift compared to the \#2 to \#7 vertical, observed under superheating. That means that these spectra (which are taken along the interface itself, with part of the beam sampling the occluding quartz) are not recording a specific signal when probing the interface itself. Therefore, we are lead to conclude that the interface has a minor influence on the stable liquid with respect to that exerted with the superheated liquid. Yet, the IR profile along the interface is still different from that of the bulk liquid, especially visible on the first sub-band of the $\mathrm{OH}$-stretching absorption. Therefore, the stable liquid is blue-shifted at the interface compared to the bulk, but less than the superheated liquid is.

The examination of the data taken with the sample 2 appears more contrasted. We can separate the spectra into two groups. First are those with the same IR profile as the bulk-like under superheating: the \#7 and 8 spectra for the whole band, the \#6, \#11, and \#12, for the second and third sub-bands, are the references. The crossing behaviour between the two water states (superheated/stable) establishes that the two series can be fully compared, and gives ground that the spectral difference have real physical significance. The second series gathers all the other spectra which show a spectral shift reverse to the previously-observed: this is a red shift, especially pronounced on the first sub-band and when along the interface (\#1 to \#4, \#9). We propose that this opposite effect takes place due to water-air interfaces coming now into play with the presence of vapour bubbles in the cavity. Actually the main bubble appears to the bottom of the photo (Fig. 2), but we have already suspected that rings of tiny bubbles are invisibly located along the water-solid interface, as also mentioned in the literature ${ }^{11}$.

The red-shifting must be measured with respect to the bulk-like liquid (see for instance, \#6 or $\# 7 ; \Delta \omega=10-15 \mathrm{~cm}^{-1}$ ), and not compared to the superheated liquid, itself blue-shifted. Explicitly, we assume that the liquid recorded at these locations (\#6 to \#8) where the band looks like the superheated bands, behaves with the less influence from the water-air 
interfaces. All the other spectra, without exception, show a red-shift from this reference. The red-shift indicates that the bonded $3250 \mathrm{~cm}^{-1}$ component increases at the expense of the $3650 \mathrm{~cm}^{-1}$, so that the water in this area seems more structured than the bulk. In other words, the water-air interface promotes the tetrahedrally-networked molecules: this interface is structure-maker (kosmotrope effect), to use the same framework of reasoning as above.

The present finding is consistent with the Sum Frequency Generation studies of the liquid-air interface ${ }^{26}$ which demonstrate an enrichment in 3150 and $3400 \mathrm{~cm}^{-1}$ peaks in the interfacial layer (and also in the $3700 \mathrm{~cm}^{-1}$, but for the topmost layer). It was additionally showed ${ }^{26}$ that the structured peak component significantly increases when inorganic ions concentrate at the interface. Thus, we cannot exclude that silica (or other) ions dissolved from the host or trapped during the synthesis procedure, would have concentrated at the interface highlighting the kosmotrope effect.

\subsubsection{Main conclusions}

As a whole, our IR study gives grounds that the superheated and the stable liquid have the same features in terms of molecular networking, which establish an interesting experimental basis to calculate the thermodynamic properties of superheated water by extrapolating its usual EoS, calibrated on the stable liquid.

Furthermore, the two liquids seem to act differently when occluded in a solid cavity, in terms of their interfacial dynamics. We are not able to propose a clear view of the supporting microphysical mechanisms, but we got evidences that the interfacial signature is enforced along the superheated water-solid interfaces. Even if we cannot propose any explanations, we may outline that this fact could be the first direct reason (to our knowledge) of why the superheating is so efficient in fluid inclusions with respect to any other systems, a stillpending question ${ }^{12}$.

But again, the main surprise is our ability to record an unambiguous signal while the physicochemical interfacial effects are classically expected to be some molecular layers thick. Actually, the inward unbalanced forces which make up the specificity of the interfacial region 
are globally of the van der Waals type and so cannot extend far away the immediate neighbour layers. We may only speculate on a long-range interaction afforded by dissolved impurities introduced either during the procedure or by dissolution of the host solid. In the literature, the finite size effects making up the "confinement" have recently been proposed to extend over up to $50 \mathrm{~nm}$ (slowing dynamics ${ }^{28}$ ), or even $320 \mathrm{~nm}$ (far- and mid-infrared spectroscopy ${ }^{29}$ ).

\section{CONCLUSION}

The first evidence obtained through this study is that the superheated water probed during this experiment is bulk water, in the sense of its intermolecular arrangement. The direct consequence of such evidence is that the properties of superheated water may be predicted using the equation of state derived to describe the "normal" water, at least down to the -80 MPa here attained. That result confirms previous observations ${ }^{8}$ which are easily overlooked since the authors did not stress their importance on this regard. Extrapolating the equation of state at $(\mathrm{P}-\mathrm{T})$ pairs results in the thermodynamic features of the metastable water, as assumed more or less empirically by previous authors ${ }^{13,15-16}$.

The IR signal shows that the water state varies from location to location in a hydraulically continuous volume, depending on the interfacial effects and the local geometries. Water-solid and water-air interfaces showed opposite effect in terms of the water structure: the former blue-shifts the signal, which we summed up (certainly too simply) as a structure-breaker effect, and the latter red-shifts the $\mathrm{OH}$-stretching band (a structure-maker feature), two findings which meet others conclusions from the literature, but cannot be generalized since they obviously depend strongly on the experimental materials and the local conditions.

These effects can play a role on the liquid water behavior when occluded liquid invades all the volume (monophasic inclusion) and becomes superheated. In particular, it seems that the molecular networking at the water-solid interfaces could be instrumental under superheating to stabilize the occluded liquid towards the nucleation. However, the measurements gained 
throughout this study are not sufficient to establish a firm conclusion on the exact role played by the interfaces to stabilize the entrapped liquid. It is noteworthy to stress that we are not able to separate the potential mixed effects between the interfacial role and the confinement, possibly acting also in solids in the thinnest part $\left(50-500 \mathrm{~nm}^{28,29}\right)$ of the cavities. In parallel, we recorded an IR signature attributed to the water-air interface which appears pretty simple to interpret, at least qualitatively: the structure-maker effect along the bubble frontier should add an additional energy cost to the usual surface tension to be paid to form any interfacial area. This is a well-known fact embedded in the Classical Nucleation Theory.

At last, we must state that the initial question is still pending and more work remains to be performed to completely address this issue of the excess stability of water in fluid inclusions as well the IR ability to record the interfacial signatures. However, the IR spectroscopy appears to be a promising tool when used at high resolution on these special samples. We confirm the surprising ability of vibrational spectroscopy to get insights the $\mathrm{H}$-bond networking at the sub-wavelength scale. Le Caer et al. ${ }^{29}$ observed the effects of confinement over hundreds of nanometers, and also were able to record the appearance of liquid-air interfaces during drying experiments. The present work is a further encouraging step in the same direction, to use the IR micro-spectroscopy in studying the versatility of the liquid water properties.

In terms of perspective, the first track is to get a precise tri-dimensional image of the cavity, and the second one to record the IR signals as a function of the size of the air bubble which can be easily controlled by the temperature.

Acknowledgements - This work has received financial support from the French Agency for Research (Agence Nationale de la Recherche), grant SURCHAUF-JC05-48942, and benefited from the SOLEIL beamtime allocation $n^{\circ} 20080054$. The authors thank C. Ramboz (ISTO, France) who lent them the sample used throughout this study. Two anonymous reviewers are acknowledged for their constructive comments, of great help to improve the initial manuscript. 


\section{REFERENCES}

${ }^{1}$ Wei C., Steudle E., and Tyree M.T. Trends Plant Sci. 4(9), 372 (1999).

${ }^{2}$ Cochard H. C.R. Physique 7, 1018 (2006).

${ }^{3}$ McManus K.J. and Davis R.O. Géotechnique 47(1), 173 (1997).

${ }^{4}$ Setzer M.J. J. Colloid Interf. Sci. 243, 193 (2001).

${ }^{5}$ Or D. and Tuller M. Water Resour. Res. 38(5), 1061, (2002).

${ }^{6}$ Pettenati M., Mercury L., and Azaroual M. Applied Geochem. 23, 3799 (2008).

${ }^{7}$ Gawin D. and Sanavia L. Transp. Porous Media 81, 141 (2010).

${ }^{8}$ Green J.L., Durben D.J., Wolf G.H. and Angell C.A. Science 249, 649 (1990).

${ }^{9}$ Zheng Q., Durben D.J., Wolf G.H. and Angell C.A. Science 254, 829 (1991).

${ }^{10}$ Alvarenga A.D., Grimsditch M., and Bodnar R.J. J. Chem. Phys. 98, 11, 8392 (1993).

${ }^{11}$ Shmulovich, K. Mercury L., Thiéry R., Ramboz C., and El Mekki M. Geochim. Cosmochim. Acta, 73(9), 2457 (2009).

${ }^{12}$ Caupin F. and Herbert E. C.R. Phys. 6, 1000 (2006).

${ }^{13}$ Wagner W. and Pruss A. J. Phys. Chem. Ref. Data 31, 2, 387 (2002).

${ }^{14}$ Brubach J.-B., Mermet A., Filabozzi A., Gerschel A., and Roy P. J. Chem. Phys. 122, art. 184509 (2005).

${ }^{15}$ Span R. and Wagner W. Int. J. Thermophys. 18, 6, 1415 (1997).

${ }^{16}$ Mercury, L., Azaroual, M., Zeyen, H., Tardy, Y. Geochim. Cosmochim. Acta. 67, 1769 (2003).

${ }^{17}$ Van Thiel M., Becker E.D., and Pimentel G.C. J. Chem. Phys. 27, 486 (1957).

${ }^{18}$ Crupi V., Majolino D., Longo F., Migliardo P., and Venuti V. Vibrational Spectros. 42, 375 (2006).

${ }^{19}$ Crupi V., Majolino D., Migliardo P., Venuti V. and Bellissent-Funel M.-C. Mol. Phys. 101(22), 3323 (2003).

${ }^{20}$ Crupi V., Majolino D., Migliardo P. and Venuti V. J. Mol. Liq. 117, 165 (2005).

${ }^{21}$ Attard P. Adv. Colloid Interf. Sci. 104, 75 (2003). 
Revised version of the Manuscript ID CP-ART-10-2011-023221. Submitted to Physical Chemistry Chemical Physics. December 14 $4^{\mathrm{h}}, 2011$.

${ }^{22}$ Doshi D.A., Watkins E.B., Israelachvili J.N. and Majewski J. PNAS 102(27), 9458 (2005).

${ }^{23}$ Benesi A.J., Grutzeck M.W., O’Hare B., and Phair J.W. J. Phys. Chem. B 2004, 108, 17783 (2004).

${ }^{24}$ Manciu M. and Ruckenstein E. J. Colloid Interf. Sci. 304, 541 (2006).

${ }^{25}$ Leroy P., Lassin A., Azaroual M., and André L. Geochim. Cosmochim. Acta 74, 5427 (2010).

${ }^{26}$ Shultz M.J., Baldelli S., Schnitzer C., and Simonelli D. In Water in confining geometries, edited by V. Buch \& J.P. Devlin (Springer Verlag, Berlin, Germany, 2003).

${ }^{27}$ Roedder E. Science 155, 1413 (1967).

${ }^{28}$ Musat R., Renault J.-P., Candelaresi M., Palmer J., Le Caër S., Righini R., and Pommeret S. Angew. Chem. Int. Ed. Engl. 47, 8033 (2008).

${ }^{29}$ Le Caër S., Pin S., Esnouf S., Raffy Q., Renault J. Ph., Brubach J.-B., Creff G., Roy P. (2011) Phys. Chem. Chem. Phys. 13, 17658-17666.

${ }^{30}$ Famin V., Nakashima S., Jolivet L., Philippot P. Contrib Mineral Petrol 146, 736-749 (2004). 